\title{
EFFECTS OF EXERCISE ON FREE SERUM CHOLESTEROL
}

\author{
D. L. MONTGOMERY, Ph.D.* and A. H. ISMAIL, Ph.D. \\ Department of Physical Education, \\ Purdue University, West Lafayette, \\ Indiana, 47907 U.S.A.
}

\begin{abstract}
Two age groups (young and old, $n=12$ ) matched for physical fitness and two physical fitness groups (high and old, $n=12$ ) matched for age participated in a four month physical fitness programme. Blood samples were drawn at four stages of metabolic stress at the pre-test and five stages at the post-test. The blood samples were analyzed by colorimetric methods for total cholesterol and free cholesterol. Statistical analysis revealed that: (1) Short-term exercise increased total cholesterol, free cholesterol, and the percent free cholesterol from the resting state to the submaximal and maximal exercise states. (2) There was no change in free serum cholesterol from the pre- to post-tests. (3) The high-fit group, compared with the low-fit group, had a lower free cholesterol level but had similar percent free cholesterol values. (4) There was no significant difference between age groups for either total serum cholesterol, free cholesterol, or the percent free cholesterol levels.
\end{abstract}

\section{INTRODUCTION}

The association of hyperlipidemia with the atherosclerotic process is well documented in a wide range of experimental animals (Clarkson, 1963) and in man (Friedman, 1969). With increasing cholesterol concentration, there is an increased risk of coronary heart disease. There is no evidence of a critical level of serum cholesterol which separates high from low risk individuals.

Compared with other species, man has a high concentration of total cholesterol (Goodman, 1965). The percentage of free cholesterol usually does not change significantly even when the total cholesterol level is considerably altered (Goodman, 1965). Free cholesterol makes up about one-third of the total serum cholesterol, while the remaining two-thirds is esterified with fatty acids (Goodman, 1965). Since free cholesterol is more atherogenic than cholesterol ester (Lacko et al, 1972), the percentage of free serum cholesterol in conjunction with the total cholesterol concentration is an important factor in the development of atherosclerosis.

It appears that physical activity will reduce serum cholesterol levels when there is a corresponding decrease in the percentage of body fat weight (Campbell, 1966, 1968; Montoye et al, 1959; Tooshi, 1971). Also, habitually active populations have lower serum cholesterol levels than sedentary populations (Casdorph, 1972; Faulkner et al, 1969; Karvonen et al, 1961; Lowenstein, 1964).

The purpose of the present investigation was to observe the effect of a four-month programme on the total, free, and percent free serum cholesterol levels in high and low fitness groups matched for age and weight, and in young and old age groups matched for physical fitness and weight.

\section{METHODS}

Twenty-four men ranging in age from 24 to 65 years, were selected on the basis of age, weight and physical fitness level from 100 men who participated in the physical fitness programme at Purdue University, consisting of calisthenics, jogging, and recreational activities such as basketball, volleyball, swimming, squash, and handball. The $\mathbf{2 4}$ men were selected so that there was a young group $(n=12)$ and an older group $(n=12)$ which were matched for weight and physical fitness. In addition, the same 24 men were divided into two physical fitness groups using the test criterion of Ismail et al, (1965), a 'low-fit' group and a 'high-fit' group ( $n=12)$. The two fitness groups were matched for age and weight. All subjects had clinically normal serum glucose: 70 to $120 \mathrm{mg} \%$ (Harleco), and triglyceride: 30 to $200 \mathrm{mg} \%$ (Oxford), levels.

The subjects were tested at the beginning and at the end of the four-month fitness programme. Venous blood samples were drawn at four stages of metabolic stress: rest, submaximal exercise $(10$ minutes of treadmill walking at 3 m.p.h. with the elevation increased by $2 \%$ every 2 minutes), maximal exercise (running on a treadmill at 6 m.p.h. with the elevation increased by $2 \%$ every 2 minutes), and recovery, (15 minutes of rest following the treadmill run). On the post-test, two blood samples were drawn during maximal exercise, with the first

*Present Address for correspondence: David L. Montgomery, (Assistant Professor), Dept. of Physical Education, McGill University, 475 Pine Avenue West, Montreal, Quebec, H2W 1S4, Canada. 
blood sample taken at the same stress that corresponded to the pre-test. The second blood sample was obtained to reflect the new maximal level.

The biochemical variables were measured by colorimetric methods. Serum triglycerides were measured according to Oxford Laboratories Procedure." Serum glucose levels were measured according to a Harleco Procedure. * The total cholesterol and free cholesterol concentrations were measured by the Hycel Method."

Data were analyzed by a factorial analysis of variance with subjects grouped by age-fitness groups. The Newman-Keuls analysis was used for mean comparison whenever the analysis of variance results yielded significance at a minimum of 0.10 level.

\section{RESULTS}

The means and standard errors of the variables used to select the age and fitness groups are presented in

* Tri-glyceride determination, 'Tri-Chol Principle', Oxford Laboratories, Foster City, Cal.

* * Glucose determination, Harleco Inc., Philadelphia, Pen.

*** Cholesterol determination, Hycel Inc., Houston, Tex.
Table I. The young and old groups were matched fo weight and physical fitness. The high-fit and low-fio groups were matched for weight and age. Both age. groups and both fitness groups improved significantly i physical fitness level from the pre-to the post-tests. The glucose levels were similar for both the young and ol groups and the high-fit and low-fit groups. There was significant difference in resting serum triglyceride levele्ల between the high-fit and low-fit groups.

The means and standard errors of the total serumb cholesterol, free cholesterol, and percent free cholesterol for the two age groups and the two fitness groups aren presented in Tables II, III and IV, respectively. The percent free cholesterol values were obtained by:

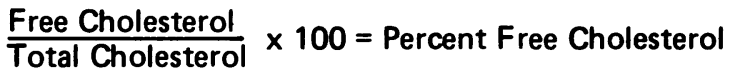

For the three biochemical variables, a pattern is shown starting with a resting mean value that increases during submaximal exercise, followed by further increasesduring maximal exercise and decreases during the 15 minute recovery period.

Table $V$ shows the analysis of variance for the serum total cholesterol, free cholesterol and percent free cho-

TABLE I

Pre-and Post-Programme Test Values ( $\bar{X}$ and S.E) of the Selection Variables for the Age and Fitness Groups $(n=12)$

Selection Variable

Age (yrs.) Pre Test

Physical Fitness Score

Pre-Test

Post-Test

$\mathrm{t}$

Weight $(\mathrm{kg})$

Pre-Test

Post-Test

$\mathbf{t}$

Resting Serum Glucose (mg\%)

$$
\begin{aligned}
& \text { Pre-Test } \\
& \text { Post-Test }
\end{aligned}
$$

$\mathrm{t}$

Resting Serum Triglyceride (mg\%)

$$
\begin{aligned}
& \text { Pre-Test } \\
& \text { Post-Test }
\end{aligned}
$$$$
t
$$

Age
Young
$36.8 \pm 1.7$
$351.7 \pm 15.5$
$374.3 \pm 13.0$
$4.04^{*}$

$80.8 \pm 3.2$

$80.0 \pm 3.0$

1.33
$89.8 \pm 1.2$
$89.3 \pm 2.1$
0.24

$123.6 \pm 20.8$

$112.6 \pm 13.2$

0.67
Old

$52.9 \pm 1.3$
$76.3 \pm 2.9$

- 1.62

$$
\begin{gathered}
91.4 \pm 2.5 \\
91.1 \pm 2.6 \\
0.09
\end{gathered}
$$

$125.8 \pm 19.6$
$122.1 \pm 16.0$
0.42
$404.9 \pm 9.8$

$420.7 \pm 8.0$

3.33*

$77.7 \pm 3.0$
$77.2 \pm 3.0$
0.93

Fitn
High-Fit
$44.4 \pm 3.1$

$404.9 \pm 9.8$
$420.7 \pm 8.0$
$3.33^{*}$

$77.7 \pm 3.0$
$77.2 \pm 3.0$
0.93

$89.5 \pm 2.1$
$88.6 \pm 1.7$
0.30

$90.3 \pm 7.7$
$92.8 \pm 8.1$
0.30

Fitness Groups
Low-Fit

$45.3 \pm 2.6 \frac{\mathrm{c}}{\mathrm{w}}$

क


TABLE //

Pre-and Post-Programme Values ( $\bar{X}$ and S.E.) of the Serum Total Cholesterol at Selected Metabolic Levels for the Age and Fitness Groups $(n=12)$

Motabolic Level

Pre Test

Rest

Submax

Max 1

Rec

Post Test

Rest

Submax

Max 1

Max II

Rec

\begin{tabular}{ll}
\multicolumn{2}{c}{ Age Groups } \\
Young & \multicolumn{2}{l}{ Old } \\
$242.7 \pm 17.1$ & $230.8 \pm 8.1$ \\
$253.4 \pm 17.0$ & $240.4 \pm 8.8$ \\
$280.4 \pm 17.8$ & $262.0 \pm 9.0$ \\
$248.1 \pm 16.5$ & $230.8 \pm 9.0$ \\
& \\
$234.1 \pm 20.0$ & $229.8 \pm 8.8$ \\
$252.8 \pm 21.5$ & $247.4 \pm 9.0$ \\
$276.1 \pm 22.7$ & $271.0 \pm 9.9$ \\
$281.0 \pm 22.4$ & $271.5 \pm 9.8$ \\
$246.3 \pm 19.8$ & $244.3 \pm 8.1$
\end{tabular}

Fitness Groups
High-Fit

$218.5 \pm 9.4$

$229.7 \pm 9.8$

$257.6 \pm 8.9$

$222.8 \pm 8.9$

$213.8 \pm 9.5$

$235.9 \pm 11.3$

$262.6 \pm 11.4$

$264.7 \pm 11.4$

$231.8 \pm 9.9$
$255.0 \pm 14.7$

$264.2 \pm 14.9$

$284.8 \pm 17.3$

$256.1 \pm 15.5$

$250.1 \pm 18.1$

$264.3 \pm 19.5$

$284.5 \pm 21.5$

$287.8 \pm 21.1$

$258.7 \pm 18.1$

TABLE III

Pre- and Post-Programme Values ( $\bar{X}$ and S.E) of the Free Serum Cholesterol at selected metabolic levels for the Age and Fitness Groups $(n=12)$

Metabolic Level

Pre Test

$\begin{array}{lll}\text { Rest } & 59.0 \pm 3.9 & 54.3 \pm 2.7 \\ \text { Submax } & 61.8 \pm 4.0 & 60.0 \pm 2.9 \\ \text { Max I } & 69.3 \pm 4.2 & 67.8 \pm 3.6 \\ \text { Rec } & 60.8 \pm 4.5 & 58.3 \pm 3.2\end{array}$

Post Test

$\begin{array}{lll}\text { Rest } & 55.1 \pm 5.1 & 52.8 \pm 2.2 \\ \text { Submax } & 61.5 \pm 5.9 & 59.6 \pm 2.6 \\ \text { Max I } & 69.0 \pm 6.2 & 65.8 \pm 2.5 \\ \text { Max II } & 68.4 \pm 6.1 & 66.2 \pm 2.0 \\ \text { Rec } & 59.1 \pm 5.8 & 58.3 \pm 1.7\end{array}$

Age Groups

$\begin{array}{rr}\text { Age Groups } \\ \text { Young } & \text { Old }\end{array}$

Fitness Groups

High-Fit Low-Fit

$51.6 \pm 2.5$

$55.0 \pm 2.7$

$62.9 \pm 2.6$

$54.5 \pm 2.4$

$61.7 \pm 3.5$
$66.8 \pm 3.3$
$74.3 \pm 4.3$
$64.7 \pm 4.5$

$49.3 \pm 3.1$

$56.7 \pm 3.4$

$63.9 \pm 3.4$

$63.8 \pm 3.3$

$54.9 \pm 3.1$

TABLE IV

Pre-and Post-Programme Values ( $\bar{X}$ and S.E) of the Percent Free Serum Cholesterol at selected metabolic levels for the Age and Fitness Groups ( $n=12)$

Metabolic Level

Pre Test

Rest

Submax

Max 1

Rec

Post Test

Rest

Submax

Max 1

Max II

Rec

$\begin{array}{rr}\text { Age Groups } \\ \text { Young } & \text { Old }\end{array}$

$24.5 \pm 0.7$

$24.6 \pm 1.0$

$25.0 \pm 0.9$

$24.6 \pm 0.9$

$23.3 \pm 0.6$

$24.1 \pm 0.8$

$24.9 \pm 0.8$

$24.2 \pm 0.7$

$23.9 \pm 0.8$
$23.5 \pm 0.8$
$25.0 \pm 0.8$
$25.8 \pm 0.9$
$25.2 \pm 0.8$

$23.0 \pm 0.6$

$24.0 \pm 0.4$

$24.3 \pm 0.6$

$24.5 \pm 0.6$

$23.9 \pm 0.5$
Fitness Groups

High Fit
$23.6 \pm 0.6$
$24.1 \pm 0.9$
$24.5 \pm 0.7$
$24.6 \pm 0.9$

$22.8 \pm 0.7$
$23.9 \pm 0.7$
$24.3 \pm 0.7$
$24.1 \pm 0.7$
$23.6 \pm 0.6$

Low-Fit

$24.3 \pm 0.8$

$25.5 \pm 0.8$

$26.3 \pm 1.1$

$25.3 \pm 0.8$

$23.5 \pm 0.6$

$24.2 \pm 0.6$

$24.9 \pm 0.6$

$24.7 \pm 0.6$

$24.3 \pm 0.7$ 
lesterol. The ANOVA technique was used to determine the significant changes, if any, between fitness groups (high versus low), between age groups (young versus old) between tests (pre versus post), between metabolic stages (rest, submaximal, maximal and recovery), and between the interactions of the various factors. For each of the three biochemical variables, two ANOVA tables are presented in Table $V$. The first ANOVA table utilizes the maximal I level of exercise, while the second ANOVA table utilizes the maximal II level of exercise. On the pre-test, there was only maximal I exercise, whereas on the post-test, maximal I exercise corresponded to the same exercise level as in the pre-test, while maximal II exercise indicated the new maximal level. At the post-test, maximal I exercise actually represented a high submaximal exercise level. It is necessary to present two ANOVA tables for each biochemical variable so that the degrees of freedom for the $F$ tests would not be fictitious as would be the case if five metabolic levels were used and maximal I was repeated in the pre-test to correspond with maximal 11 in the post-test.

For total chlesterol, the effect of metabolic levels was significant at the 0.01 level. There was a significant increase in serum total cholesterol from the resting state of the submaximal exercise, and a further significant increase during maximal exercise with a significant decrease during the recovery period. The interaction of tests by metabolic stages (TM) was significant at the 0.10 level. In the post-test, the resting serum total cho lesterol was lower than at the pre-test whereas at af. other stages of exercise, the post-test total cholesterotconcentration was higher than the pre-test total cho? lesterol concentration.

The interaction of fitness groups by metabolic stage $\frac{5}{8}$ (FM) was significant at the 0.10 level using the meta bolic stages that included maximal I exercise but was nof significant using the metabolic stages that included maxis mal II exercise. The significant interaction using maxis mal I exercise but insignificant interaction using max $\overrightarrow{\mid \omega}$ mal II exercise was possibly related to the amount of time run on the treadmill by the high and low fitness. groups. The longer the time run on the treadmill, the more time that is allowed for lipids to be mobilized from the tissues. Since the high-fit group compared to the low-fit groups (15.40 min. versus $5.67 \mathrm{~min}$.) ran for longer time on the treadmill during maximal I exercise is comparison with maximal II exercise (17.88 $\mathrm{min}$. versus. 10.11 min.), the mean differences between fitness groups were more apparent in FM using maximal I thare in FM using maximal II exercise. The differences in serum total cholesterol between the low-fit group and the high-fit group were smaller during maximal I exercise in comparison with the other metabolic stages, whicto resulted in a significant interaction at maximal $I$ exercises in Table $V$. The total cholesterol responses at maximaß

TABLE V

Analysis of Variance for Serum Total Cholesterol, Free Cholesterol, and Percent Free Cholesterol

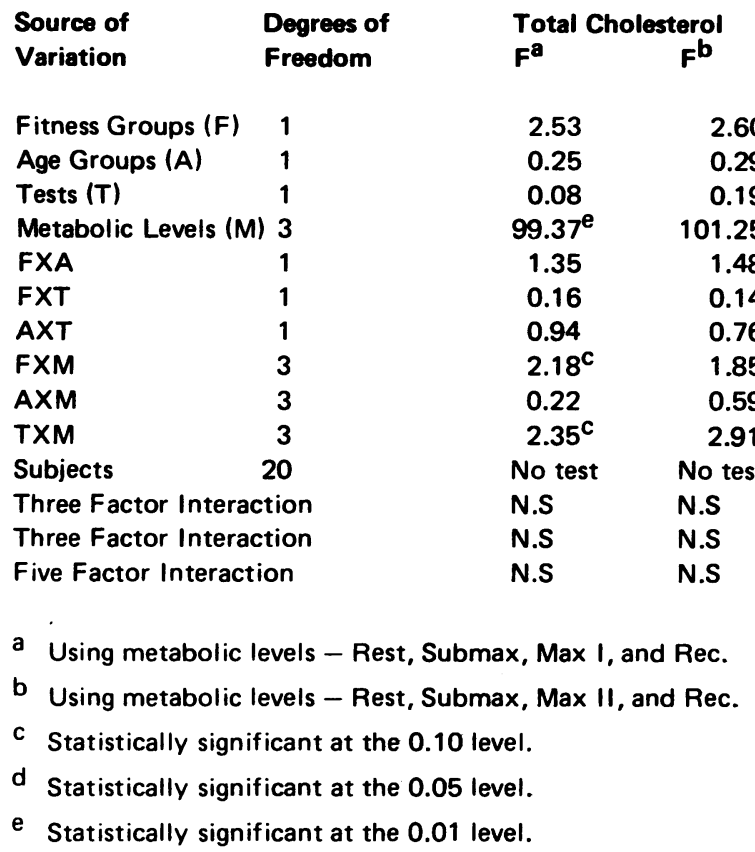

\begin{tabular}{|c|c|c|c|}
\hline \multicolumn{2}{|c|}{ Free Cholesterol } & \multicolumn{2}{|c|}{ \% Free Cholesterol } \\
\hline$F^{a}$ & $\mathbf{F}^{\mathbf{b}}$ & $F^{\mathbf{a}}$ & $\mathbf{F}^{\mathbf{b}}$ \\
\hline $3.71^{\mathrm{C}}$ & $3.87^{C}$ & 1.43 & 1.46 \\
\hline 0.23 & 0.22 & 0.01 & 0.02 \\
\hline 0.40 & 0.39 & 1.56 & 1.88 \\
\hline $76.07^{e}$ & $65.71^{e}$ & $7.96^{\mathrm{d}}$ & $6.11^{d}$ \\
\hline 1.44 & 1.50 & 0.06 & 0.02 \\
\hline 0.52 & 0.48 & 0.21 & 0.22 \\
\hline 0.02 & 0.04 & 0.10 & 0.03 \\
\hline 0.14 & 0.11 & 0.33 & 0.29 \\
\hline 0.48 & 0.45 & 1.01 & 1.31 \\
\hline 0.84 & 0.86 & 0.09 & 0.12 \\
\hline No test & No test & No test & No test \\
\hline N.S & N.S & N.S & N.S. \\
\hline N.S & N.S & N.S & N.S \\
\hline N.S & N.S & N.S & N.S \\
\hline
\end{tabular}


exercise were dependent upon the fitness level which determined the subjects' tolerance to stress.

The effect of metabolic levels was significant at the 0.01 level for free serum cholesterol and at the 0.05 level for percent free cholesterol. There was a significant increase in free cholesterol in the serum from the resting concentration to the submaximal exercise, with a further increase during maximal exercise, and a significant decrease during the recovery period. The percent free cholesterol at rest was significantly lower than the percent free cholesterol during exercise and during the recovery period. Acute exercise increased both the absolute and the percent free cholesterol. The high-fit group in comparison to the low-fit group had a significantly $(P<$ 0.10) lower free cholesterol. However, there was no significant difference in percent free cholesterol between the two fitness groups.

\section{DISCUSSION}

Long duration exercise can alter the serum cholesterol level because cholesterol can be degraded by the oxidation of its side chajn to carbon dioxide (Chaikoff et al, 1952; Myant \& Lewis, 1966). Side chain oxidation of cholesterol is increased during exercise in man (Malinow \& Perley, 1969) and in rats (Malinow et al, 1968). There is some evidence that serum cholesterol levels can be reduced by decreasing body weight (Campbell, 1966, 1968; Faulkner et al, 1969; Lowenstein, 1964; Mock, 1972; Montoye et al, 1959; Teraslinna, 1966; Watt et al, 1972; Zauner, 1970). Generally, in overweight individuals there is a loss in body weight as a result of a long-term exercise programme so that exercise may indirectly be associated with the decrease in serum total cholesterol level. Since there was no significant reduction in body weight in the 24 subjects in this study, this finding could explain why there was no significant change in serum total cholesterol concentration from the pre- to post-tests.

In this study the high-fit group, in comparison to the low-fit group, had a significantly lower free cholesterol concentration. In the design of the study, the two fitness groups were matched for weights, however there was a significant difference between the high-fit and low-fit groups in terms of the percentage lean body weight. At pre-test, the high-fit group was $86.3 \%$ lean and the low-fit group was $80.0 \%$ lean. Montoye et al, (1974) have reported a significant positive correlation between total cholesterol and body fatness as measured by the sum of four skinfolds in 4000 subjects aged 10 to 64 years. Thus, the difference in percent lean body weight between the two fitness groups may have contributed to the significant difference between the two groups. Habitual exercise over several years by the high-fit group could possibly explain the differences.
In man, the lecithin-cholesterol acyltransferase (LCAT) enzyme catalyzes the transfer of fatty acids from the $\beta$-position of lecithin to free cholesterol in the plasma (Glomset, 1962; Glomset et al, 1962; Sperry, 1935). Since human plasma does not contain enzymes that hydrolyze cholesterol esters, the esterification of free cholesterol represents the activity of LCAT.

The effect of short-term exercise in this study, increased the total cholesterol, free cholesterol and percent free cholesterol. During the recovery period, the total cholesterol and both the absolute and percent free cholesterol decreased. Since there was an increase in total lipid mobilization including total cholesterol mobilization during exericse, it was expected that the free cholesterol level would increase as well. Since the percent free cholesterol level increased significantly, it was indirect evidence that the enzyme LCAT was unable to esterify the free cholesterol at the same rate that the free cholesterol was mobilized. A study by Abdulla et al, (1969) has noted that free cholesterol is mobilized faster than saturated or mono-unsaturated cholesterol esters which supports the results of this study.

Several studies have indicated that the LCAT esterifying activity decreases with age (Gherondache, 1963; Wagner \& Poindexter, 1952). Thus a greater ratio of esterified cholesterol has been found in younger persons than older individuals (Gherondache, 1963; Lopez et al, 1967; Wagner \& Poindexter, 1952). In the present study, there was no significant difference in percent free cholesterol between the young and old groups. Since the groups were matched for weight and physical fitness, it is possible this may account for conflict with the literature. ing:

In summary, the data in this study suggest the follow-

(1) Short-term physical exercise increased the total cholesterol and both the absolute and percent free cholesterol from the resting state to the submaximal exercise state, to the maximal exercise state, and decreased in the recovery period.

(2) There was no significant change in free serum cholesterol level due to a four-month physical fitness programme which did not alter the body weight.

(3) The high-fit group had a significantly lower free cholesterol level than the low-fit group but had similar percent free cholesterol values.

(4) There was no significant difference between age groups matched for physical fitness and weight for the serum total cholesterol, free cholesterol or percent free cholesterol levels. 


\section{REFERENCES}

Abdulla, Y. H., Adams, C. W. M., \& Morgan , R. S., 1969. Differential resorption rates of subcutaneous implants of ${ }^{3}$ i cholesterol, various ${ }^{3} \mathrm{H}$ cholesterol esters and ${ }^{3} \mathrm{H}$ cholesterol- $\left(1-{ }^{14} \mathrm{C}\right)$ linolenate, J.Atherosclerosis Res., 9, 81-85.

Barnicot, N. A., Bennett, F. J., Woodburn, J. C., Pilkington, T. R. E. \& Antonic, A., 1972. Blood pressure and serur cholesterol in the Hadza of Tanzania, Hum.Biol., 44, 87-116.

Campbell, D. E., 1966. Influences of diet and physical activity on blood serum cholesterol of young menç Am.J.Clin.Nutrn., 18, 79-85.

Campbell, D. E., 1968. Effect of controlled running on serum cholesterol of young adult males of varying morphological constitutions, Res. Quart. 39, 47-53.

Casdorph, H. R., 1972. Normal limits for serum-cholesterol, Lancet, (i), 1076.

Chaikoff, I. L., Siperstein, M. D., Dauben, W. G., Bradlow, H. L., Eastham, J. F., Tomkins, G. M., Meier, J. R., Chen, RW., Hotta, S. \& Srere, P. A., 1952. $C^{14}$-cholesterol. II. Oxidation of.carbons 4 and 26 to carbon dioxide by the intact rat, J.Biol.Chem., 194, 413-416.

Clarkson, T. B., 1963. Atherosclerosis - Spontaneous and induced, Adv. Lipid Res., 1, 211-252.

Faulkner, J. A., Montoye, H. J. \& Greey, G. W., 1969. A comparison of executives with a total population in physicate activity and other possible coronary heart disease risk factors, Med.Sci.Sports, 1, 160-164.

Friedman, M., 1969. Pathogenesis of Coronary Artery Disease, p. 269, Mc-Graw-Hill, New York.

Gherondache, C. N., 1963. Physiologic variations in the cholesterol esterifying activity of serum, J.Clin Endocrinol.Metab., 23, 1024-1028.

Glomset, J. A., 1962. The mechanism of the plasma cholesterol esterification reaction: plasma fatty acid transferase $\overline{\bar{O}}$ Biochem.Biophys.Acta, 65, 128-135.

Glomset, J. A., Parker, F., Tjaden, M. \& Williams, R. H., 1962. The esterification in vitro of free cholesterol in humañ and rat plasma, Biochem. Biophys. Acta, 58, 398-406.

Goodman, D. S., 1965. Cholesterol ester metabolism, Physiol. Rev., 45, 747-839.

Ismail, A. H., Falls, H. B. \& MacLeod, D. F., 1965. Development of a criterion for physical fitness tests from factori analysis results, J.Appl.Physiol., 20, 991-999.

Karvonen, M. J., Pekkarinen, M., Metsala, P. \& Rautonen, Y., 1961. Diet and serum cholesterol of lumberjacks Br.J.Nutrn., 15, 157-164.

Lacko, A. G., Rutenberg, H. L. \& Soloff, L. A., 1972. Reduced rate of plasma cholesterol esterification in patients with $\hat{N}^{-}$ coronary heart disease, Fed.Proc., 31, 291, (abstract). Lopez, A. S., Krehl, W. A. \& Hodges, R. E., 1967. Relationship between total cholesterol and cholesteryl esters with
age in human blood plasma, Am.J.Clin.Nutrn. 20, 808-815.

Lowenstein, F. W., 1964. Epidemiologic investigations in relation to diet in groups who show little atherosclerosis and $\cong$ are almost free of coronary ischemic heart disease, Am.J.Clin.Nutrn., 15, 175-186.

Malinow, M. R. \& Perley, A., 1969. The effect of physical exercise on cholesterol degradation in man, J.Atherosclerosis Res., 10, 107-111.

Malinow, M. R., McLaughlin, P. \& Perley, A., 1968. Cholesterol: treadmill activity accelerates oxidation in rats, Science, 160, 1239-1240. 
Mock, G. W., 1972. The effects of a four-month physical fitness program on selected free fatty acids, Doctoral Thesis, Purdue University.

Montoye, H. J., Block, W. D., Keller, J. B. \& Willis, P. W., 1974. Fitness, fatness and serum cholesterol: Epidemiologic study in a total community, Abstracts of Research Papers 1974 AAHPER Convention, p. 56.

Montoye, H. J., Van Huss, W. D., Brewer, W. D., Jones, E. M., OhIson, M. A., Mahoney, E. \& Olson, H., 1959. The effects of exercise on blood cholesterol in middle-aged men, Am.J.Clin.Nutrn., 7, 139-145.

Myant, N. B. \& Lewis, B., 1966. Estimation of the rate of breakdown of cholesterol in man by measurement of ${ }^{14} \mathrm{C}_{2}$ excretion after intravenous $\left(26-{ }^{14} \mathrm{C}\right)$ cholesterol, Clin.Sci., 30, 117-127.

Report of inter-Society Commission for Heart Disease Resources 1970. Primary prevention of the atherosclerotic diseases, Circulation, 42, A55-A88.

Sperry, W. M., 1935. Cholesterol esterase in blood, J.Biol.Chem., 111, 467-478.

Teraslinna, P., 1966. Effect of exercise on selected serum lipids and their relationships to certain variables of body structure and function, Doctoral Thesis, Purdue University.

Tooshi, A., 1971. Effects of three different durations of endurance exercise upon serum cholesterol, Med.Sci.Sports, 3, i, (abstract).

Wagner, A. \& Poindexter, C. A., 1952. Esterification of serum cholesterol. I. Serial determination in health, J. Lab.Clin.Med., 40, 321-323.

Watt, E. W., Foss, M. L. \& Block, W. D., 1972. Effects of training and detraining on the distribution of cholesterol, triglycerides, and nitrogen in tissues of albino rats, Circln. Res., 31, 908-914.

Zauner, C. W. \& Swenson, E. W., 1970. Exercise, diet and other factors on blood lipids, J.Florida Med. Assocn. 57, 30-35. 